\title{
Benthic biodiversity in the South Orkney Islands Southern Shelf Marine Protected Area
}

\author{
Brasier, M.J. ${ }^{\mathrm{a}, \mathrm{b}}$, Grant, S.M. ${ }^{\mathrm{c}}$, Trathan, P.N. ${ }^{\mathrm{c}}$, Allcock, L. ${ }^{\mathrm{d}}$, Ashford, O. ${ }^{\mathrm{e}}$, \\ Blagbrough, H. ${ }^{\mathrm{c}}$, Brandt, A. ${ }^{\mathrm{f}}$, Danis, B. ${ }^{\mathrm{g}}$, Downey, R. ${ }^{\mathrm{h}}$, Eléaume, M. P. ${ }^{\mathrm{i}}$, \\ Enderlein, P. ${ }^{\mathrm{c}}$, Ghiglione, C. ${ }^{\mathrm{j}}$, Hogg, O. ${ }^{\mathrm{c}}$, Linse, K. ${ }^{\mathrm{c}}$, Mackenzie, M. ${ }^{\mathrm{k}}$, \\ Moreau, C. ${ }^{\mathrm{g}}$, Robinson, L. ${ }^{1}$, Rodriguez, E. ${ }^{\mathrm{m}}$, Spiridonov, V. ${ }^{\mathrm{n}}$, Tate, A. ${ }^{\mathrm{c}}$, \\ Taylor, M. ${ }^{\circ}$, Waller, C. ${ }^{\mathrm{p}}$, Wiklund, $\mathrm{H}^{\mathrm{b}}{ }^{\mathrm{a}}$ and Griffiths, H.J. ${ }^{\mathrm{c}^{*}}$ \\ ${ }^{a}$ School of Environmental Science, University of Liverpool, Liverpool, UK; ${ }^{b}$ Life \\ Sciences, Natural History Museum, London, UK; ${ }^{c}$ British Antarctic Survey, Natural \\ Environment Research Council, High Cross, Cambridge, UK; ${ }^{d}$ School of Natural \\ Sciences and Ryan Institute, University of Galway, Galway, UK, ${ }^{e}$ Scripps Institution of \\ Oceanography, University of California, San Diego, USA; ${ }^{f}$ Department of Marine \\ Zoology, Senckenbegr Research Institute, Germany; ${ }^{g}$ Department of Biology of \\ Organisms, University of Brussels, Belgium; ${ }^{h}$ Fenner School of Environment \& Society, \\ Australian National University, Canberra, Australia; ${ }^{i}$ Department Origins and \\ Evolution, National Museum of Natural History, Paris, France, ${ }^{j}$ Italian National \\ Antarctic Museum, Genoa, Italy; ${ }^{k}$ Marine Invertebrates, Museums Victoria, Melbourne, \\ Australia; ${ }^{l}$ School of Earth Sciences, University of Bristol, Bristol, UK; ${ }^{m}$ Invertebrate \\ Zoology, American Museum of Natural History, New York, USA; ${ }^{n}$ P.P. Shirshov \\ Institute of Oceanology, Moscow, Russia; ${ }^{\circ}$ School of Biological Sciences, Univesrsity of \\ Essex, Essex, UK; ${ }^{p}$ Centre for Environmental and Marine Science, University of Hull, \\ Hull, UK. \\ *corresponding author.m.brasier@liverpool.ac.uk
}




\title{
Benthic biodiversity in the South Orkney Islands Southern Shelf Marine Protected Area
}

\begin{abstract}
The South Orkney Islands Southern Shelf (SOISS) Marine Protected Area (MPA) was the first MPA anywhere in the world to be designated entirely within the High Seas and is managed under the Commission for the Conservation of Antarctic Marine Living Resources (CCAMLR). As part of efforts to undertake research and monitoring in and around the MPA, an international expedition ('SO-AntEco') was undertaken in the austral summer of 2016, on board the British Antarctic Survey research ship RRS James Clark Ross. The focus of the expedition was to contribute towards a better understanding of selected benthic habitats around the South Orkney Islands, and the biodiversity within those habitats, in relation to the geomorphic zones both inside and outside the SOISS MPA. This addresses a key objective set out by the draft SOISS MPA Research and Monitoring Plan (SC-CAMLR, 2014). The benthic assemblages of the SOISS MPA region were found to be strongly correlated with the texture of the seafloor, where hard substrates hosted a greater number of individuals, taxa and biomass with a dominance of filter feeding Vulnerable Marine Ecosystem (VME) taxa, and soft sediments were dominated mostly by deposit feeders. Substantial differences in the abundance of VME taxa were found between two sampling gears used (shallow underwater camera system and Agassiz trawl). We conclude that camera systems may be more suitable for VME assessments than the Agassiz trawl, but in addition where possible, additional trawling is advisable for collecting all faunal types and for higher taxonomic resolution. The designation of VME locations or MPAs based purely on geomorphic classification is not advisable, due to small scale variation in substrate and other local physical influences; however, the utility of such classifications may be improved with the inclusion of additional environmental factors e.g. substrate type.
\end{abstract}

Keywords: Southern Ocean, Antarctica, benthos, protected area, vulnerable marine ecosystems 


\section{Introduction}

The South Orkney Islands is a small archipelago located in the Southern Ocean, 375 miles north-east of the tip of the Antarctic Peninsula. The island chain is bounded by two current regimes, to the north by the southern boundary of the Antarctic Circumpolar Current and to the south by the Weddell Sea Gyre. The seafloor around the South Orkney Islands has been shown to be an area with exceptionally high biodiversity. The marine animals there represent approximately one fifth of all species recorded for the entire Southern Ocean (Barnes et al. 2009).

The Commission for the Conservation of Antarctic Marine Living Resources (CCAMLR) is an instrument of the Antarctic Treaty System, responsible for fisheries management and the conservation of marine ecosystems in the Southern Ocean. In 2009, CCAMLR established the South Orkney Islands Southern Shelf Marine Protected Area (SOISS MPA) (CCAMLR 2009a), the first MPA located entirely within the High Seas anywhere on the planet (Figure 1). The SOISS MPA covers an area of $94,000 \mathrm{~km}^{2}$, in which all types of commercial fishing are prohibited. The MPA is designed to contribute towards the conservation of biodiversity in the South Orkney Islands region, and to protect representative examples of a range of pelagic and benthic habitats.

CCAMLR has agreed a general framework for establishing MPAs in the Southern Ocean (CCAMLR 2011), which includes the requirement to develop MPA Research and Monitoring Plans, setting out activities relevant to evaluating the achievement of specific MPA objectives. As further MPAs are established in the Southern Ocean, and existing areas are reviewed, new approaches for assessing biodiversity across such areas will be increasingly important. CCAMLR also provides for the protection of Vulnerable Marine Ecosystems (VMEs), as part of a management framework designed to avoid significant adverse impacts on benthic habitats from bottom fisheries. (CCAMLR $2009 \mathrm{~b}$ ). The frameworks uses the biomass of VME taxa, taxa deemed vulnerable to the impacts of destructive fishing methods including reef building and slow growing species for details see CAMMLR (2009c), to define 'VME Risk Areas'. A VME Risk Area will contains a minimum threshold of $10 \mathrm{~kg}$ biomass of VME taxa is recovered from a single longline segment (a $1200 \mathrm{~m}$ section of longline gear). Once identified, such Risk Areas are closed to fishing until management actions are determined by the Commission (CCAMLR 2009b).

Previous works using trawl data to describe the benthic faunal assemblages on shelf $(<$ $500 \mathrm{~m}$ depth) and slope (up to $800 \mathrm{~m}$ deep) regions around South Orkneys include Lockhart et al. (2009) and Jones and Lockhart (2011). Geographic structuring in invertebrate densities was recorded, with the highest densities clustered on the western and eastern tips of the island chain with abundance in benthic communities decreasing towards the outer limits of the shelf (Lockhart et al. 2009). In most sites sampled the 10 $\mathrm{kg}$ per $1200 \mathrm{~m}^{2}$ (assuming a $1 \mathrm{~m}$ transect of length $1.2 \mathrm{~km}$ ) threshold was exceeded and a total of 17 VME taxa were recorded dominated by Porifera, Ascidiacea, Bryozoa and Cnidaria.

SO-AntEco (JR15005) was a British Antarctic Survey (BAS) led expedition undertaken in conjunction with an international team of scientists from the Scientific Committee for Antarctic Research (SCAR) AntEco research programme. The team included 
participants from nine different countries and 16 institutes. The expedition took place on board the BAS research ship RRS James Clark Ross in the austral summer of 2016.

The focus of the SO-AntEco cruise was to contribute towards a better understanding of selected benthic habitats, and the biodiversity within those habitats, in relation to the geomorphic zones both inside and outside the MPA. This addresses a key objective set out by the draft SOISS MPA Research and Monitoring Plan (SC-CAMLR 2014). Our results from targeted sampling both inside and outside the MPA will assist in determining whether the existing geomorphic classification for this region (Dickens et al. 2014) provides a reasonable proxy for benthic communities. Information on species abundance (including VME species) and diversity with physical habitat along the shelf break will also help improve understanding of habitats both inside and outside the MPA. This is an important contribution to the understanding of benthic habitats around the South Orkney Islands, which will be relevant for the review and ongoing management of this MPA, as well as for further spatial planning in this region and potentially further afield.

\begin{abstract}
Aims
- To describe the benthic habitats and fauna within the South Orkney region.

- To investigate patterns between the physical environmental and benthic communities including; region, depth, substrate, geomorphic classification.

- To document the presence and abundance of VME species with regard to the current MPA.

- To discuss how different sampling techniques e.g. camera systems vs trawling gear can be used to describe and assess the vulnerability of different habitats.
\end{abstract}

\title{
Methods
}

\section{Data collection}

The JR15005 cruise sampled five regions within the South Orkney Islands, at depth ranges between 500 and $2000 \mathrm{~m}$. Two sites, West and South, were within the SOISS MPA and the remaining three were outside of the MPA; North West (including the North West Trough), North and North East. Prior to the deployment of sampling gears multibeam bathymetry data were collected to survey each area. A number of Shallow Underwater Camera System (SUCS) and Agassiz Trawl (AGT) transects were conducted at each of the five locations (Figure 2, Table 1).

\section{Shallow Underwater Camera System (SUCS)}

The SUCS was deployed at $500 \mathrm{~m}, 750 \mathrm{~m}$ and just shallower than $1000 \mathrm{~m}$ depth (the latter being its max operating depth). Normal protocol involved three consecutive photo transects, the direction of which was determined by wind direction (to allow the ship to sit comfortably using dynamic positioning) and seafloor topography, each transect was separated by $100 \mathrm{~m}$, with each complete transect consisting of 10 photos, themselves 
each $10 \mathrm{~m}$ apart. In some cases the three transects were not completed because of deteriorating weather conditions or icebergs in close vicinity to the ship.

\section{Agassiz Trawl (AGT)}

The AGT was used to sample animals approximately $1 \mathrm{~cm}$ and larger in length, which comprise the larger macro- and megafauna, but it did capture some smaller animals as well. Weather and topography permitting, each station comprised three replicate trawls at shallower depths $(500 \mathrm{~m}, 750 \mathrm{~m}$, and $1000 \mathrm{~m})$ and at least one trawl at the deeper depths $(1500 \mathrm{~m}$ and $2000 \mathrm{~m})$. In total, there were 75 AGT deployments over the five regions sampled (Table 1). The Agassiz trawl had a mesh size of $1 \mathrm{~cm}$ and a mouth width of $2 \mathrm{~m}$, and once on the seafloor the net was then trawled at 1 knot for 10 minutes.

On board, samples were photographed as total catch and hand-sorted into groups varying from Phylum to species level collections. The wet-weight (biomass) of the different taxa was assessed by using calibrated scales (with accuracy and resolution atsea of $0.001 \mathrm{~kg}$ ). Animals were either preserved in $96 \%$ ethanol, RNA later, $4 \%$ formalin or frozen at $-20^{\circ} \mathrm{C}$ or $-80^{\circ} \mathrm{C}$ depending on taxonomic group and the requirements of the collaborating scientists.

\section{SUCS Image analysis}

The SUCS images were used to estimate faunal density, biomass and species abundance of the benthos. Fauna were identified to different taxonomic resolutions dependant on VME status and diversity in Antarctic regions and, the resolution of the images. The size of taxa was also recorded in most cases to allow for more accurate biomass estimates where; small $\leq 3 \mathrm{~cm}$, medium $=3-6 \mathrm{~cm}$, large $\geq 6 \mathrm{~cm}$ (Table 2). Some biological material was unidentifiable to phyla but distinguishable as VME species e.g. branched or budding fragments which could be bryozoan or cnidarian species. These individuals were recorded as "VME unknown". The percentage cover of encrusting species was also recorded as the number of individuals can be uncertain with neighbouring colonies and it was not always possible to distinguish between Porifera and Bryozoa. Blind checks of photos from each event were carried out to check the accuracy of the recorded abundance values.

Weights for each size class were determined from the wet weight recorded on board during the sorting of the AGT catch. For each taxa all recorded weights were divided into size fractions and each was averaged to obtain a weight for small, medium and large cohorts. For taxa in which size classes were not used e.g. Decapoda an average individual weight was calculated from all recorded weights.

\section{Physical environment}

To allow for the analysis of potential patterns between the physical environment and faunal communities each SUCS image was assigned a texture; hard or soft, as well as the substrate; silt, dropstones (rocks and boulders deposited from melting icebergs), biogenic, boulders, rock and gravel (Supplementary Table 1). Based on their position, each SUCS set was allocated a geomorphic classification from those defined in the bathymetric complication of the South Orkney Islands region in Dickens et al. (2014) 
(Figure 2). For the AGT deployments, the texture was classified based on the corresponding SUCS data for their set. If SUCS data were not available (i.e. depths greater than $1000 \mathrm{~m}$ ) texture was determined from video data collected from a camera mounted on an epibenthic sledge (EBS) which was also deployed at each AGT location and the catch images from each AGT deployment. As substrate was variable within SUCS sets the AGT were not assigned substrate classifications, furthermore if the corresponding SUCS set contained a mixture of hard and soft substrates the AGT data were assigned a 'mixed' texture.

\section{Data analysis}

For graphical purposes faunal counts from each SUCS image were combined and averaged for each set as the number of transects and photos was not consistent due to sampling constraints such as sea state. The same method was used to calculate a comparable average wet weight per photo for each set. This wet weight was used to calculate faunal biomass over $1200 \mathrm{~m}^{2}$ (distance used by CCAMLR to define VME Risk Areas) from the known area of the SUCS field of view $\left(0.51 \mathrm{~m}^{2}\right)$. Where; weight over $1200 \mathrm{~m}^{2}=($ Average weight $* 1.961) * 1200$.

As the towed distance of each Agassiz deployment varied, the counts and wet weights recorded were standardised to $1200 \mathrm{~m}^{2}$. Based on deployment location each Agassiz trawl was assigned the same set as its closest SUCS deployment to compare faunal assemblage and gear selectivity within the same regions. Trawl data were also averaged by set to investigate variability within regions.

For statistical analysis all data from each individual photo or trawl within each set was used to investigate the relationship between faunal composition and physical variables (i.e. region, depth, substrate, texture and geomorophic classification). These were first tested using PRIMER 6 multivariate software (Primer 6 and Permanova +, Primer-E Ltd., U.K.). Bray-Curtis similarity was performed on non-transformed, species presence and abundance data. The similarity matrix was then used for cluster analysis, multidimensional scaling (MDS) and analysis of similarities (ANOSIM). Based on the $R$ statistic from ANOSIM, two-way analysis of variance (ANOVA) was used to statically determine the influence of texture, region and depth on the number of individuals and taxa as well as interactive effects between the variables on the SUCS and AGT data. All two-way ANOVA analyses were performed in R. To investigate differences in diversity between sites which may be relate to MDS groups taxa diversity for each SUCS and AGT set were calculated using formulas for Margalef's richness, Pielou's eveness, Shannon diversity (log base e) and Simpson diversity (1- $\left.\lambda^{\prime}\right)$.

At present, VMEs are mostly detected or documented by commercial fishing vessels the majority of which use hooked longline gears. Despite their potential high abundances, small or encrusting VME taxa are unlikely to be collected and recorded using these methods. We investigate the abundance of "large" ( $\geq 6 \mathrm{~cm})$ VME taxa, those most likely to be collected by commercial fishing gear, to consider the potential differences in our observations to those of fishing activities. 


\section{Results}

\section{Physical characteristics of the seafloor}

The geomorphic classification of the South Orkneys in Dickens et al. (2014) demonstrates the bathymetric complexity of this region and potential for different habitats within the sampled locations. The sites sampled covered several geomorphological units including the shelf, gentle and steep slopes, valleys and troughs as well as flatter regions including such as plateaus. Each of the five regions sampled contained multiple geomorphological units and some sets were positioned across two geomorphic classifications (Figure 2).

A combination of SUCS imagery, EBS video and photos of the trawl catch were used to determine the substrate characteristics of each SUCS transect and trawl location. The dominant substrate determined by SUCS imagery varied both within and between regions. With the exception of two sets the western regions, (North West, North West Trough and West), were dominated by soft silt substrates covering 74 to $100 \%$ of each transect (Figure 2). All other regions; North, North East and Southern, were characterised by hard substrates with nine out of eleven sets dominated by rock (53 to $87 \%$ coverage), while soft silt substrates were only recorded in three of these eleven sites accounting for 7 to $34 \%$ coverage. Biogenic substrates were only recorded in one set, S_1000a, where $42 \%$ of the seafloor was covered in dead barnacle material. Dropstones were only recorded on soft substrates in the North West and West sites, NW_750a and W_750a (they might exist on harder substrates elsewhere but would be impossible to visually distinguish from local rocks). Boulders were found in regions dominated by hard substrates and were most common in the S_750a set at 38\%.

\section{Benthic assemblages from SUCS data}

A total of 31 taxa from 10 known phyla were identified from the SUCS imagery (Table 2). By number of individuals, most regions and sets were dominated by "VME unknown" taxa, echinoderms and cnidarians with frequent byozoan and Porfiera taxa. Overall, out of the 11945 individuals counted 32.03\% were unknown VME taxa and the highest identified taxa were brittle stars accounting for $19.5 \%$ of individuals. The highest total number of individuals from VME taxa from a single site was recorded at the Southern set, S_750a, with $118.63 \pm 33.30$ individuals per photo (Figure 3a), comprised mostly of unknown VME taxa. Both 500 and $1000 \mathrm{~m}$ sets within the Southern region had relatively high abundance of individuals as well as $500 \mathrm{~m}$ sets within the North West (59.03 \pm 21.9 individuals $)$ and the North East $(33.46 \pm 22.51$ individuals). The lowest abundances were recorded in the North West set deeper than $500 \mathrm{~m}$, all depths within the West and the lowest being the North West trough with $1 \pm$ 0.89 individuals per photo.

By wet weight Porifera dominated sets in the Southern, North East and North regions. In the western regions, whilst Porifera contributed to substantial proportions of wet weight within some sets in the North West, eight out of the 10 sets in the North West and West were dominated by echinoderm and cnidarian taxa. Average wet weight by region mostly reflected the abundance patterns described however the greatest wet weights within these regions did not always correlate with abundance (Figure $3 b$ ). For example the greatest wet weight per photo within the Southern region was recorded at 
$500 \mathrm{~m}(0.67 \mathrm{~kg})$, followed by $1000 \mathrm{~m}(0.60 \mathrm{~kg})$ and $750 \mathrm{~m}(0.45 \mathrm{~kg})$. These weights were dominated by Porifera accounting for 65 to $91 \%$ of the total wet weight. Within the Northern sites the overall biomass per set reflected the abundance data, with greatest biomass recorded at the $500 \mathrm{~m}$ sites North East ' $a$ ' and North West ' $b$ ' sets. Again the North West and West sites had the lower recorded wet weights, the lowest at the West $750 \mathrm{~m}$ site at $0.006 \mathrm{~kg}$ per photo.

\section{Benthic assemblages from AGT data}

The regional differences recorded in the number and wet weight of benthic taxa recorded using AGT did not mirror those of the SUCS data. The Southern region which dominated the in both number of individuals and wet weight for SUCS had some of the lowest abundances from, on average, $471(500 \mathrm{~m})$ to $12(2000 \mathrm{~m})$ individuals and wet weight at $2.64(500 \mathrm{~m})$ to $0.76 \mathrm{~kg}(1500 \mathrm{~m})$ (Figure 4). Both the number of individuals and wet weight obtained from AGT deployments were highest for the NW_500a site where an abundance of annelid worms were collected, accounting for $62 \%$ of the individuals and $48 \%$ of the biomass. Annelids were also a substantial part of the fauna collected at the N_500a (68\% of individuals) and NW_750a (58\% of individuals) sets. Again echinoderms were dominant for both numbers and wet weight at many locations in the Northern regions. As recorded by SUCS the Porifera dominated faunal biomass in the North East and Southern regions as well as the West. A decline in the number of individuals and biomass with depth was observed in the majority of regions except the North East where there was an abundance of echinoderms and Porifera at the NE_1000a and NE_2000b locations.

\section{Relationship with environmental variables}

\section{SUCS}

The relationships between the physical factors and faunal assemblage was variable (Figure 5). The ANOSIM results indicated that region, depth, geomorphic zone and substrate had limited correlation with faunal composition as measured by SUCS data, (Supplementary Table 2 ). However texture explained $0.661 \%$ of the variation in similarity. The $R$ statistics and significance levels for pairwise comparisons within the large scale physical groups, geomophology and region, were reflected this. These results were greater in pairwise comparisons between regions or geomorphic zones where one group was dominated by hard textures and the other by soft textures, (Supplementary Table 2). For example the West and South comparison with where $R=$ 0.706 and $\mathrm{P}=0.1 \%$ the west was dominated by soft substrates and the south by hard.

Two-way ANOVA was used to test for interactive effect of region and depth with texture (Table 3). For the SUCS data, depth did not have a significant effect, reflecting the low ANOSIM $R$ value and the limited depth range sampled. Interactive effects between texture and region were recorded for both the number of individuals and taxa. On average hard substrates were inhabited by nearly 3 times as many taxa and 15 times as many individuals as soft substrates. Furthermore, with the exception of Simpson's all diversity measures were significantly higher for hard substrates (Figure 6). 
In addition to the observed differences in abundance and diversity, the faunal composition was also different between texture types. Hard textured areas were dominated by VME unknown and encrusting taxa accounting for $51 \%$ of individuals, for soft substrates these taxa combined accounted for $8 \%$ of individuals (Figure 7). Instead textured areas were, on average, dominated by the echinoderms, more specifically Holohturoidea (35\%), Ophiuroidea (20\%) and Echinoidea (5\%). Both textures had similar relative abundances of identified Porifera, Byozoa and different cnidarian taxa. Soft substrates had higher relative abundances of more mobile taxa such as Arthropods including decapod and mysid shrimps as well as fish.

$A G T$

As the AGT data were collected over a great depth range, 0 to $2000 \mathrm{~m}$, this provided an opportunity to re-examine potential associations between faunal composition and depth following the decline in the total number of individuals and wet weight observed in most regions (Figure 4). Depth was shown to have a significant effect on the number of individuals and taxa present (Table 3). Additionally, region also had a significant effect on the number of individuals and interactive effects between texture and depth and, texture and region were shown. On average the number of taxa and individuals declined from 17.5 to 9.71 and 1506.3 to 70.58 respectively between 500 and $2000 \mathrm{~m}$ depth. This trend was reflected in the abundance of several taxa including Annelids, Pynogonida, Bryozoa, Ascidacea, Cnidarians, Echinoderms, Molluscs and Nemertea (Supplementary Table 3). Some variation in diversity indices was recorded with depth, most noticeably all indices for $1500 \mathrm{~m}$ were lower than shallower depths (Figure 8).

\section{Distribution of VME taxa}

In total $17 \mathrm{VME}$ taxonomic categories were observed from SUCS imagery and AGT material. The standardised wet weight, to $1200 \mathrm{~m}^{2}$, per taxa by set for each gear is displayed in Supplementary Tables 4 and 5. Some VME taxa were very rare in the data set including, brachiopods, cnidarians and the scallop Adamussium colbecki whilst some were completely absent e.g. serpulid worms.

The VME biomass estimated by SUCS greatly exceeded collected by the AGT (Figure 9). For 18 out of the 21 SUCS sets VME biomass exceed $10 \mathrm{~kg}$ per $1200 \mathrm{~m}^{2}$, the threshold set out in CCAMLR CM 22-07. As indicated by the SUCS assemblage descriptions, the Southern sites contained the greatest VME biomass with a combined total of $3906.66 \mathrm{~kg}$ over the three sets. For the AGT single set, NE_500a, exceeded the $10 \mathrm{~kg}$ threshold which was associated with a high wet weight of Porifera,

(Supplementary Table 5). A general decline in VME biomass with depth was recorded between 500 and $2000 \mathrm{~m}$ however most regions had very low biomass, $<1 \mathrm{~kg}$, at all sites including $500 \mathrm{~m}$.

Both the AGT and SUCS data clearly shows that the total VME biomass is driven by Porifera. Several taxa including cnidarians and bryozoans were, relatively well represented in the SUCS data from the Northern and Southern regions. Echinoderms represented a similar proportion of the biomass in both the North West and West regions for both AGT and SUCS. Large VME taxa were mostly dominated in numbers by cnidarian taxa with Porifera and unknown VMEs (Figure 10,11). In comparison to 
total VME the large VME taxa accounted for 41 to $97 \%$ of VME weight and $<1$ to $54 \%$ of the VME counts. This range was related to the dominance of sponges or encrusting species within each set.

\section{Discussion}

\section{Benthic assemblages and physical variables}

The influence of physical environmental variables such as location, depth and substrate on benthic assemblages has been reported for other shallow, shelf and slope regions of Antarctica. The importance of each of these variables can depend on the scale of the investigation. For example, in the coastal benthic communities of the Ross Sea the substrate type and composition explained $66 \%$ of the variation in shallow water macrofaunal communities within surveyed transects (Cumming et al. 2006). Similar results with regard to substrate and faunal composition have also been recorded at King George Island and the South Orkneys (Quartino et al. 2001, Richardson et al. 1979). In this study fine scale habitat heterogeneity associated with hard and soft textures on the seafloor explained the majority of the variation within the data. Additionally the larger scale factors region and depth were found to have interactive effects on the benthos. Similar results have been recorded in East Antarctica where dropstones were associated with significant increase in faunal abundance and diversity at the sub-metre scale (Post et al. 2017).

Our results demonstrate that the abundance, biomass and taxonomic diversity of fauna within the South Orkney region was greater on hard substrata. This corresponds with deeper shelf community investigations, c. $1000 \mathrm{~m}$, where megafaunal density is often higher in areas of coarse sediment (Jones et al. 2007, Post et al. 2011). In the Weddell Sea and the Ross Sea it has been suggested that the differences in diversity could be related to the abundance of suspension feeding taxa that generally prefer hard substrata being highly diverse within Antarctic waters (Gutt and Starmans 1998; Jones et al. 2007). The coarse resolution of our taxonomic dataset prevents us from analysing the number of such species between these sediments; however a greater diversity amongst hard substrates on the East Antarctic coast using a similar taxonomic resolution was also recorded in Post et al. (2017).

The hard substrates of the South Orkneys region were dominated by sessile filter feeding VME species including encrusting species, indeterminate VME taxa, cnidarians and Porifera. Softer substrates were dominated by holothurians. This result is not surprising given their deposit feeding traits preferentially selecting and ingesting fine substrata (Gutt 1990). Ophiuroids were also relatively dominant for both substrates albeit not to the same levels recorded in Post et al. (2017) where they contributed to $50 \%$ of the fauna for hard substrates and $20 \%$ for soft. Ophiuroids as well as other echinoderm taxa such as urchins are generally considered ubiquitous to the Southern Ocean dominating many localities and depths as they can thrive on both hard and soft substrates (Thrush et al. 2006).

A significant difference in the number of individuals with depth was recorded from the AGT data. No clear differences in the community assemblage or diversity indices were found which may be associated with a number of factors, including the taxonomic resolution of the data. At a higher taxonomic resolution (e.g. family, genus or species) 
changes in assemblage may be more evident, for example Neal et al. (2017) recorded a significant change in polychaete taxa within depths in the Scotia Arc and the Amundsen Sea. Different faunal assemblages may also have been recorded if the sampling depths had extended beyond the slope.

\section{Gear selectivity}

The differences in the fauna present, abundance and total wet weight between sets, regions and depths were not always coherent between sampling gears. Some variations might be partially associated with the variability in substrate within regions and slightly different sampling locations. However the AGT hauled lower abundances and biomass which is likely to be associated with the gears themselves.

The nature of the AGT limits the collection of small encrusting species as well as small VME taxa which would not be dislodged by the trawl as it is towed along the seafloor. Thus the SUCS imagery was able to provide more information about the presence and abundance of VME taxa than trawling alone. Previous studies by Jones and Lockhart (2011) have also noted that seafloor imagery provided direct evidence of VME habitats where, in the same location, there were insufficient taxa within trawl collections to exceed the $10 \mathrm{~kg} / 1200 \mathrm{~m}^{2}$ threshold. Taxa not represented within their trawl contents were bryozoan species, stylasterid, scleractinian and primnoid corals, similar to the taxa undersampled in trawl deployments in this study. By comparison the trawls did contain fauna that could not be identified from the SUCS including VME associated species, for example the symbiotic polychaete such as Polyeunoa laevis which live within the branches of coral species (Serpetti et al. 2016). However, we emphasize that destructive sampling methods such as trawling are not needed to document the presence and abundance of VME taxa to threshold levels and furthermore video and image analysis may be more suitable especially when sampling in vulnerable or recently disturbed localities (Bowden et al. 2005).

Both gears have limited abilities to sample infauna, however on occasion the AGT did get embedded in soft sediment collecting a larger number of infaunal species. This occurred at the NW_500a site where a large number and biomass of annelid worms were collected, skewing our trawl data. Small mobile taxa such as amphipods and mysid shrimps can be hard to distinguish in images and are better represented in the AGT collection. For these reasons, where possible multiple gears should be used to investigate the benthic diversity of these areas. As the SUCS is a downward facing camera which can only land on 'flat' substrates, certain regions could not be sampled, including steep slopes, boulders and canyons. The topography of such habitats is also unsuitable for towed gears, yet they are often considered to be diversity hotspots (Robert et al., 2015; Fernandez-Acaya et al. 2017). Such environments are thus often excluded from benthic surveys, potentially excluding diverse regions.

\section{VME taxa}

Prior to this investigation the presence and abundance of VME taxa surrounding the MPA has been studied in similar localities but over shallower depth ranges (50-800 m) than those investigated here (e.g. Lockhart and Jones 2008, Jones and Lockhart 2011). Here we find that VME taxa were most abundant in the Southern region where the MPA is currently established. Locations to the North East of the islands were still relatively 
high in VME taxa and exceeded $10 \mathrm{~kg} / 1200 \mathrm{~m}^{2}$, the CCAMLR VME threshold (CCAMLR 2009b). In contrast to previous studies, regions on the western side of the South Orkneys yielded lower abundances of VME taxa associated with the dominance of soft substrates. Additionally, with the exception of one set in the Northeast region (NE_500a), the AGT samples alone would not have been sufficient to identify VME habitats within the sampled regions. This is lower than previous investigations, at shallower depths, where eleven out of the 75 stations samples exceed the $10 \mathrm{~kg} / 1200 \mathrm{~m}^{2}$ VME trigger (Jones and Lockhart 2011).

\section{Further Comparison between the MPA and surrounding regions}

The MPA itself is located on the southern side of the South Orkney Islands, a region which in previous studies of the neighbouring shelf environments yielded a lower faunal biomass compared to the west and eastern regions (Lockhart and Jones 2008). This may be a result of the convergence of the Antarctic Circumpolar Current and the Weddell Sea Bottom Water, as well as smaller scale disturbance by iceberg scouring and historical fishing (Lockhart and Jones 2008). Thus faunal differences between the MPA and non MPA regions may have been expected. However the observed differences benthic communities within and outside the MPA vary between the SUCS and AGT. This is driven by the contrasting abundance and biomass data in the South region. The SUCS data could suggest the MPA protects one of the most productive regions sampled (South) but also incorporates less productive areas (West), explained by the regional differences in texture. The AGT data however would suggest that the MPA incorporates two of the least productive regions sampled.

Differences between the two gears has been discussed and based on the SUCS data no obvious trends between the sites inside and outside of the MPA. Differences between the regions inside and outside of the MPA may have been expected if they were experiencing different levels of disturbance from bottom fishing gears. At present the only active fisheries surrounding the South Orkney MPA are krill which are unlikely to impact the benthos (Constable 2000). With the increasing pressures for commercial demersal fisheries in the region, these data provide a useful comparison to assess potential future impacts to benthic communities.

\section{Implications for marine management}

The use of physical surrogates to predict species assemblages and VME habitats would provide a valuable tool to support conservation and management in the Southern Ocean, particularly in undersampled or vulnerable regions. For example, such models have been applied to several regions of the Antarctic shelf (e.g Beaman and Harris 2005, Thrush et al. 2006) and slope (Post et al. 2010). Within this investigation we find evidence that fine scale variation in seafloor texture may limit our ability to predict the presence and abundance of species using larger scale factors such as geomorphology, region or depth. The difficulty in predicting faunal composition based on large-scale physical factors due to the influence of small-scale physical variability is also discussed in Cummings et al. (2006).

The influence of small-scale habitat heterogeneity on the presence of VME taxa suggests that further benthic sampling is need to accurately assess the VME status of such habitats. A better understanding of this relationship is required before modelling 
approaches can be used to reliably determine management strategies based on large scale factors, such as geomorphology, alone. The relationship between benthic community composition and environmental characteristics is complex with many variables contributing to differences in community composition and the spatial structure of biodiversity (Convey et al., 2014; Post et al., 2017). Given the current international effort to establish a representative system of marine protected areas in the Southern Ocean it is important to continue investigating the relationships between both broad and local scale physical surrogates that could be used to infer VME locations and potential MPA sites. Studies have shown that when geomorphic units were used to predict benthic fauna only weak distinctions between assemblages were found; however when additional environmental factors are available a greater distinction between the benthic communities with habitat is possible (Douglas et al. 2014).

Characterisation and improved understanding of benthic habitats around the South Orkney Islands will contribute to the objectives of the SOISS MPA draft research and monitoring plan, and to the MPA review scheduled for 2019. It is also relevant for spatial planning across the wider Scotia Sea region, and to the consideration of how a range of sampling methods and gear types can be utilised to best effect in obtaining information that can be used to ensure appropriate protection for vulnerable and ecologically important areas.

\section{Conclusions}

Substantial differences in the abundance of VME taxa were found between the two sampling gears. Camera systems may be more suitable for VME assessments but where possible trawling is advisable for the collection of all faunal types for higher resolution taxonomic investigations. Despite this the benthic assemblages of the SOISS MPA region were strongly correlated with substrate, where hard substrates housed a greater number of individuals, taxa and biomass with a dominance of filter feeding VME taxa, and soft sediments were dominated by mostly deposit feeders. The lack of correlation between large scale physical factors and small-scale habitat heterogeneity can have a significant influence on the overall level and type biodiversity found a region. For this reason the designation of VME locations or MPAs based purely on geomorphic classification should not be advised, due to small scale variation in substrate and other local physical influences; however the utility of such classifications may be improved with the inclusion of additional environmental factors.

\section{Disclosure statement}

No potential conflict of interest was reported by the authors.

\section{Author contributions}

MJB identified the fauna within the SUCS imagery, prepared data for analysis and constructed this manuscript. HJG was the principal scientist on the SO-AntEco expedition and contributed to data collection, analysis and interpretation. SG, PT and KL significantly contributed to the analysis and interpretation of the data presented. MJB, SG, LA, OA, HB, AB, BD, RD, ME, PE, CG, OH, MM, CM, LR, ER, VS, AT, 
MT, CW and HW were on the SOAntEco expedition and contributed to data collection, specimen identification and the production of this manuscript.

\section{Acknowledgements}

We thank the officers and crew of the RRS James Clark Ross for their tireless efforts in supporting the scientific team during the JR15005 cruise. We also acknowledge the institutes and funders of all of the international participants from the SO-AntEco team. We would also like to thank Drs Beatrix Schlarb-Ridley and Mark Belchier for their help and support during the production of this manuscript, and Dr Peter Fretwell for the production of Figure 1.

\section{Funding}

This work is part of the British Antarctic Survey's 'Biodiversity, Evolution and Adaptation' and 'Ecosystems' core science programmes, and contributes to the SCAR AntEco scientific research programme. The NERC Collaborative Gearing Scheme aided the participation of Madeleine Brasier, Laura Robinson, Michelle Taylor and Helena Wiklund on the JR15005 cruise.

\section{References}

Barnes, D. K., Kaiser, S., Griffiths, H. J., \& Linse, K. 2009. "Marine, intertidal, freshwater and terrestrial biodiversity of an isolated polar archipelago." Journal of Biogeography, 36 (4): 756-769. doi: 10.1111/j.1365-2699.2008.02030.x.

Beaman, R.J. and Harris, P.T. 2005 "Bioregionalization of the George V Shelf, East Antarctica." Continental Shelf Research, 25 (14): 1657-1691. doi:10.106/j.csr.2005.04.013.

Bowden, D.A. 2005 "Quantitative characterization of shallow marine benthic assemblages at Ryder Bay, Adelaide Island, Antarctica.” Marine Biology, 146 (6): 1235-1249.

CCAMLR, 2009a. Conservation Measure 91-03. Protection of the South Orkney Islands Southern Shelf. Schedule of Conservation Measures in Force 2017/18. Hobart, Australia.

CCAMLR. 2009b. Conservation Measure 22-07. Interim measure for bottom fishing activities subject to Conservation Measure 22-06 encountering potential 
vulnerable marine ecosystems in the Convention Areas. Schedule of Conservation Measure in Force 2017/18. Hobart, Australia.

CCAMLR. 2009c. CCAMLR VME Taxa Classification Guide Version 2009. Hobart, Australia.

CCAMLR. 2011. Conservation Measure 91-04, General Framework for the establishment of CCAMLR Marine Protected Areas. Schedule of Conservation Measures in Force 2017/18. Hobart, Australia.

Convey, P., Chown, S. L., Clarke, A., Barnes, D. K. A., Bokhorst, S., Cummings, V., Ducklow, H. W., et al. 2014. "The spatial structure of Antarctic biodiversity." Ecological Monographs 84 (2): 203-244. doi:10.1890/12-2216.1.

Constable, A.J., de la Mare, W.K., Agnew, D.J., Everson, I. and Miller, D., 2000. Managing fisheries to conserve the Antarctic marine ecosystem: practical implementation of the Convention on the Conservation of Antarctic Marine Living Resources (CCAMLR). ICES Journal of marine Science 57 (3): 778-791. doi: doi:10.1006/jmsc.2000.0725.

Cummings, V., Thrush, S., Norkko, A., Andrew, N., Hewitt, J., Funnell, G. and Schwarz, A.M. 2006. "Accounting for local scale variability in benthos: implications for future assessments of latitudinal trends in the coastal Ross Sea." Antarctic Science, 18 (04): 633-644. doi:10.1017/S0954102006000666

Dickens, W.A., Graham, A.G., Smith, J.A., Dowdeswell, J.A., Larter, R.D., Hillenbrand, C.D., Trathan, P.N., Erik Arndt, J. and Kuhn, G. 2014 “A new bathymetric compilation for the South Orkney Islands region, Antarctic Peninsula (49-39 W to 64-59 S): Insights into the glacial development of the continental shelf." Geochemistry, Geophysics, Geosystems, 15 (6): .2494-2514. doi:10.1002/2014GC005323.

Douglass, L.L., Turner, J., Grantham, H.S., Kaiser, S., Constable, A., Nicoll, R., Raymond, B., Post, A., Brandt, A. and Beaver, D. 2014 “A hierarchical 
classification of benthic biodiversity and assessment of protected areas in the Southern Ocean.” PloS one, 9 (7): 100551. doi:10.1371/journal.pone.0100551

Fernandez-Arcaya, U., Ramirez-Llodra, E., Aguzzi, J., Allcock, A.L., Davies, J.S., Dissanayake, A., Harris, P., et al. 2017 “Ecological Role of Submarine Canyons and Need for Canyon Conservation: A Review." Frontiers in Marine Science. 4 (5). doi:10.3389/fmars.2017.00005

Gutt J., and Starmans A.1998. "Structure and biodiversity of megabenthos in the Weddell and Lazarev Seas (Antarctica): ecological role of physical parameters and biological interactions." Polar Biolgy, 20 (4): 229-247.

Jones, D.O., Bett, B.J. and Tyler, P.A. 2007. "Depth-related changes to density, diversity and structure of benthic megafaunal assemblages in the Fimbul ice shelf region, Weddell Sea, Antarctica.” Polar Biology, 30 (12): 1579-1592.

Jones, C.D. and Lockhart, S.J. 2011. "Detecting Vulnerable Marine Ecosystems in the Southern Ocean using research trawls and underwater imagery." Marine Policy, 35 (5): 732-736. doi:10.1016/j.marpol.2011.02.004.

Lockhart, S.J. and Jones, C.D. 2008. "Biogeographic patterns of the benthic invertebrate megafauna on shelf areas within the Southern Ocean Atlantic Sector." CCAMLR Science. 15: 167-192.

Lockhart, S.J., Wilson, N., Lazo-Wasem, E. and Jones, C.D. 2009. "Benthic invertebrate composition and characterisation of the South Orkney Islands." Chapter 10 in U.S. AMLR 2008/09 Field Season Report. NOAA-TM-NMFSSWFSC-445.

Neal, L., Linse, K., Brasier, M.J., Sherlock, E. and Glover, A.G. 2017. “Comparative marine biodiversity and depth zonation in the Southern Ocean: evidence from a new large polychaete dataset from Scotia and Amundsen Seas.” Marine Biodiversity. doi:10.1007/s12526-017-0735-y 
Parker, S.J. and Bowden, D.A. 2010. "Identifying taxonomic groups vulnerable to bottom longline fishing gear in the Ross Sea region." CCAMLR Science, 17:105127.

Post, A.L., O’Brien, P.E., Beaman, R.J., Riddle, M.J. and De Santis, L. 2010 'Physical controls on deep water coral communities on the George V Land slope, East Antarctica.' Antarctic Science, 22 (4): 371-378. doi:10.1017/S0954102010000180.

Post, A.L., Beaman, R.J., O’Brien, P.E., Eléaume, M. and Riddle, M.J. 2011. "Community structure and benthic habitats across the George V Shelf, East Antarctica: trends through space and time.” Deep Sea Research Part II: Topical Studies in Oceanography, 58 (1): 105-118. doi:10.1016/j.dsr2.2010.05.020.

Post, A.L., Lavoie, C., Domack, E.W., Leventer, A., Shevenell, A. and Fraser, A.D. 2017. "Environmental drivers of benthic communities and habitat heterogeneity on an East Antarctic shelf." Antarctic Science, 29 (1): 17-32. doi:10.1017/S0954102016000468.

Quartino, M., KloÈser, H., Schloss, I. and Wiencke, C. 2001. "Biomass and associations of benthic marine macroalgae from the inner Potter Cove (King George Island, Antarctica) related to depth and substrate.” Polar Biology, 24 (5): 349-355.

Richardson, M.G. 1979. "The distribution of Antarctic marine macroalgae related to depth and substrate.” British Antarctic Survey Bulletin, 49, pp. 1-13.

Robert, K., Jones, D.O.B., Tyler, P., Van Rooij, D. \& Huvenne, V.A.I. 2015. “Finding the hotspots within a biodiversity hotspot: fine-scale biological predictions within a submarine canyon using high-resolution acoustic mapping techniques." Marine Ecology 36 (4): 1256-1276. doi:10.1111/maec.12228.

SC- CAMLR. 2014. Research and Monitoring Plan for the South Orkney Islands Southern Shelf Marine Protected Area (MPA Planning Domain 1, Subarea 48.2). Paper submitted by the Delegation of the European Union to the Thirty-Third 
meeting of the Scientific Committee. SC-CAMLR-XXXIII/11. Hobart, Australia.

Serpetti, N., Taylor, M.L., Brennan, D., Green, D.H., Rogers, A.D., Paterson, G.L.J. and Narayanaswamy, B.E. 2016. "Ecological adaptations and commensal evolution of the Polynoidae (Polychaeta) in the Southwest Indian Ocean Ridge: A phylogenetic approach.” Deep Sea Research Part II: Topical Studies in Oceanography. 137: 273-281. doi:10.1016/j.dsr2.2016.06.004.

Thrush, S., Dayton, P., Cattaneo-Vietti, R., Chiantore, M., Cummings, V., Andrew, N., Hawes, I., Kim, S., Kvitek, R. and Schwarz, A.M. 2006. "Broad-scale factors influencing the biodiversity of coastal benthic communities of the Ross Sea." Deep Sea Research Part II: Topical Studies in Oceanography, 53 (8): 959-971. doi:10.1016/j.dsr2.2006.02.006.

\section{Table and figure captions}

Table 1. Number of AGT deployments and SUCS transects per set by depth and region.

Table 2. List of all taxa observed and identified from SUCS imagery collected on JR15005 including those which were recorded based on size and with VME status. From the taxonomic data collected on board from AGT samples these data were later assigned the same taxonomic resolution for these analyses.

Table 3. Table 3 Two-way ANOVA results between physical variables and the number of individuals and taxa from SUCS and AGT data. Significant values $(\mathrm{P}<0.05)$ indicated in bold.

Figure 1. Location of the South Orkney Island Shelf Shelf marine protected area.

Figure 2. Location of the SUCS and AGT deployments of the SO-AntEco expedition by region, depth and set, with geomorphic classification from Dickens et al. (2014) and percentage cover of substrate type (averaged within each SUCS set). $\mathrm{N}=$ North, NE = North East, NW $=$ North West, NWT $=$ North West Trough, $\mathrm{W}=$ West and S $=$ South. 
Figure 3. Average number of individuals (upper) and wet weight per photo $\left(0.51 \mathrm{~m}^{2}\right)$ (lower) per phylum by set. Unknown phyla include both encrusting species and unidentified VME taxa. Error bars for weight are too high to include in the plot. Box indicates sampled locations within the MPA.

Figure 4. Average number of individuals (upper) and wet weight per trawl (lower) per phylum by set. Each trawl was standardised to $1200 \mathrm{~m}^{2}$ prior to analyses. Box indicates sampled locations within the MPA.

Figure 5. MDS of region, substrate, depth and texture of SUCS species assemblages.

Figure 6. Average abundance of taxa and individuals (left) with average diversity indices (right) from SUCS imagery by texture with standard deviation.

Figure 7. Proportional abundance of taxa from SUC imagery averaged by texture. Taxa coloured by phyla, phyla with $<1 \%$ not shown.

Figure 8. Average abundance and diversity indices from AGT data by depth with standard deviation.

Figure 9. Average wet weight of VME taxa by phyla by set for both SUCS (upper) and AGT (lower). Box indicates sampled locations within the MPA.

Figure 10. The relative abundance of large VME taxa averaged by SUCS set, size of pie chart proportional to the abundance recorded.

Figure 11. The relative wet weight of large VME taxa averaged by SUCS set, size of pie chart proportional to the total wet weight recorded. 


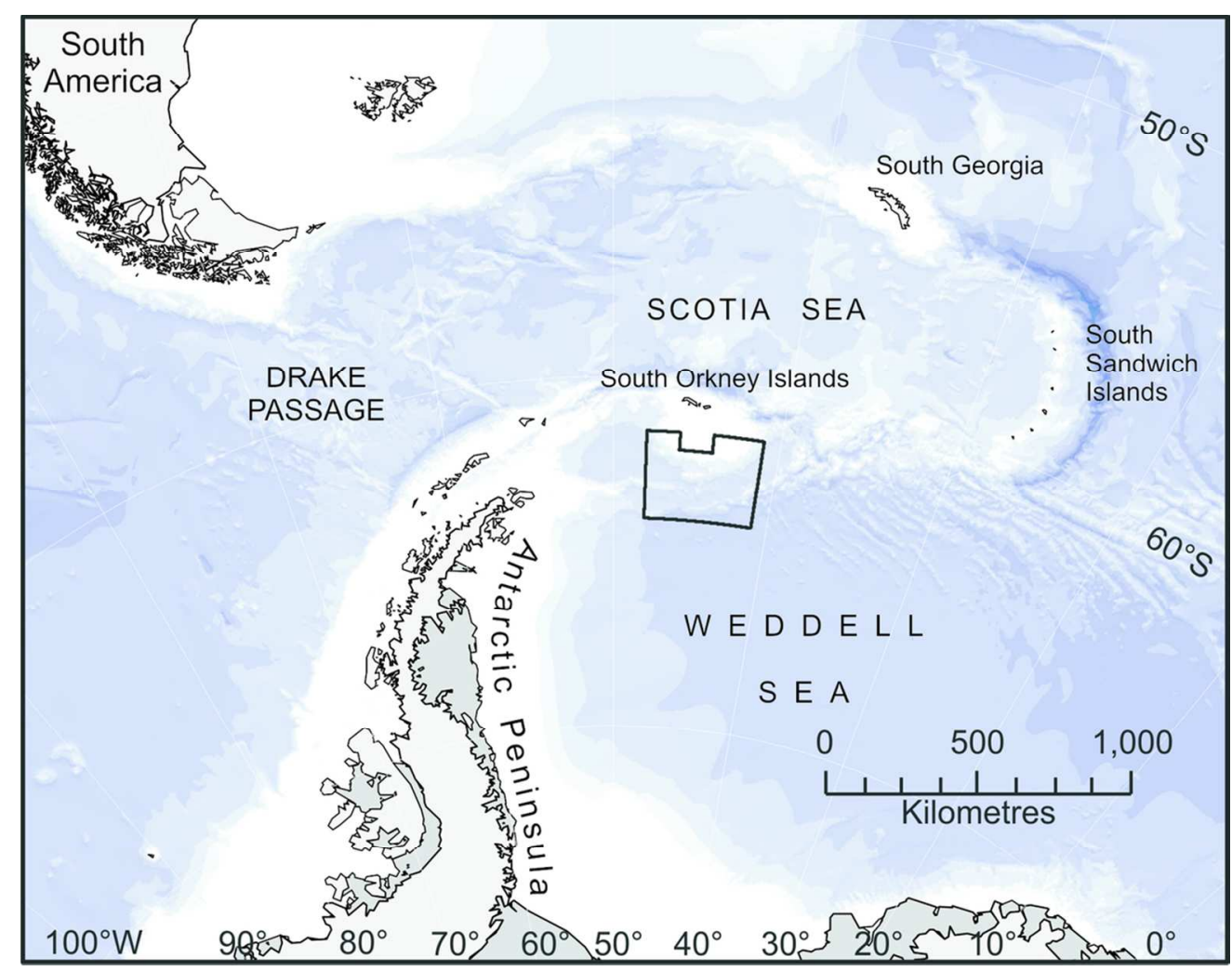

Figure 1. Location of the South Orkney Island Shelf Shelf marine protected area. $102 \times 80 \mathrm{~mm}(300 \times 300 \mathrm{DPI})$ 
Figure 2. Location of the SUCS and AGT deployments of the SO-AntEco expedition by region, depth and set, with geomorphic classification from Dickens et al. (2014) and percentage cover of substrate type (averaged within each SUCS set). $\mathrm{N}=$ North, NE = North East, NW = North West, NWT = North West Trough, W = West and $\mathrm{S}=$ South.

$329 \times 222 \mathrm{~mm}(300 \times 300 \mathrm{DPI})$ 
Figure 3. Average number of individuals (upper) and wet weight per photo $(0.51 \mathrm{~m} 2)$ (lower) per phylum by set. Unknown phyla include both encrusting species and unidentified VME taxa. Error bars for weight are too high to include in the plot. Box indicates sampled locations within the MPA.

$$
209 \times 297 \mathrm{~mm}(300 \times 300 \mathrm{DPI})
$$




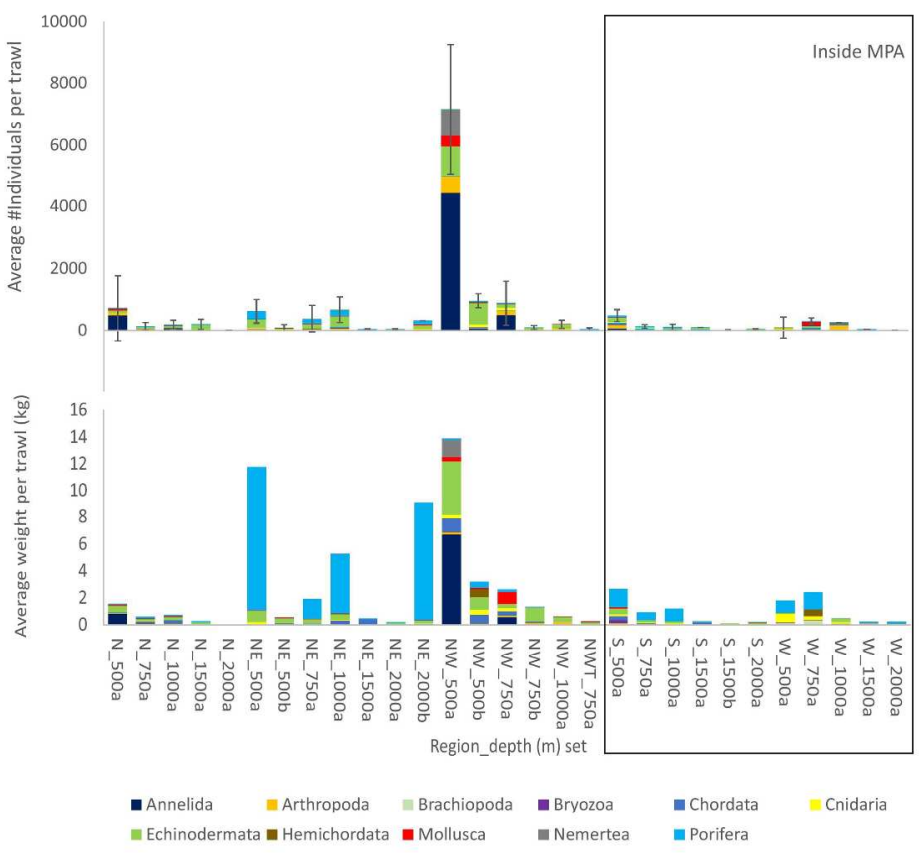

Figure 4. Average number of individuals (upper) and wet weight per trawl (lower) per phylum by set. Each trawl was standardised to $1200 \mathrm{~m} 2$ prior to analyses. Box indicates sampled locations within the MPA.

\section{$209 \times 297 \mathrm{~mm}(300 \times 300$ DPI $)$}




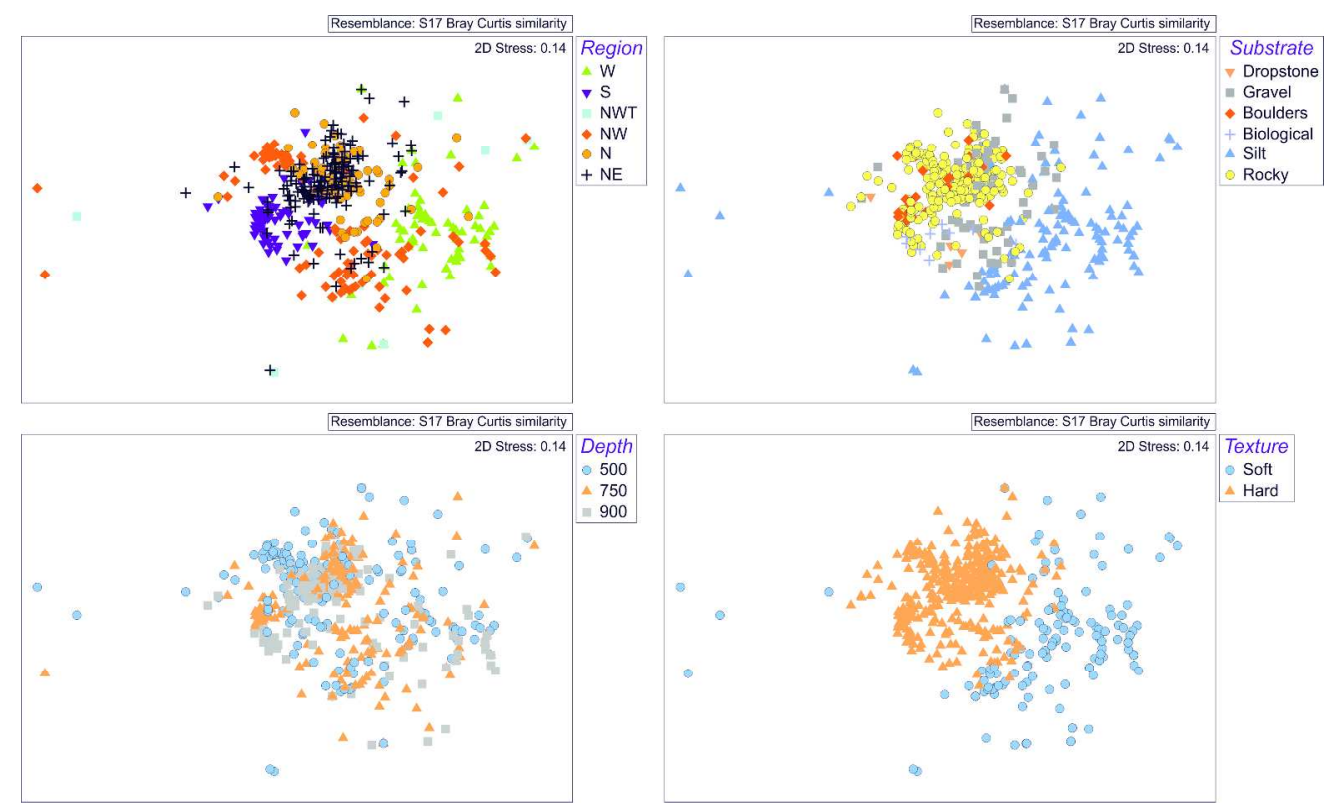

Figure 5. MDS of region, substrate, depth and texture of SUCS species assemblages.

$330 \times 200 \mathrm{~mm}(300 \times 300$ DPI $)$ 
Figure 6. Average abundance of taxa and individuals (left) with average diversity indices (right) from SUCS imagery by texture with standard deviation.

$209 \times 297 \mathrm{~mm}(300 \times 300$ DPI $)$ 
Figure 7. Proportional abundance of taxa from SUC imagery averaged by texture. Taxa coloured by phyla, phyla with $<1 \%$ not shown.

$209 \times 297 \mathrm{~mm}(300 \times 300$ DPI)

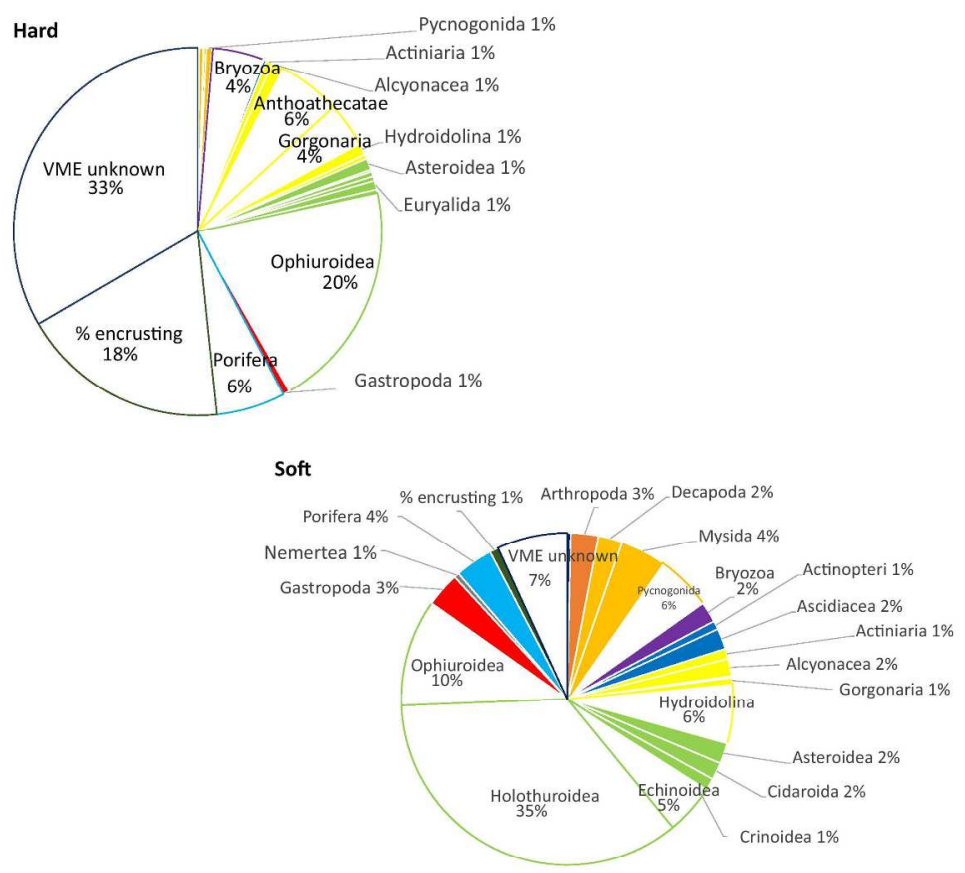



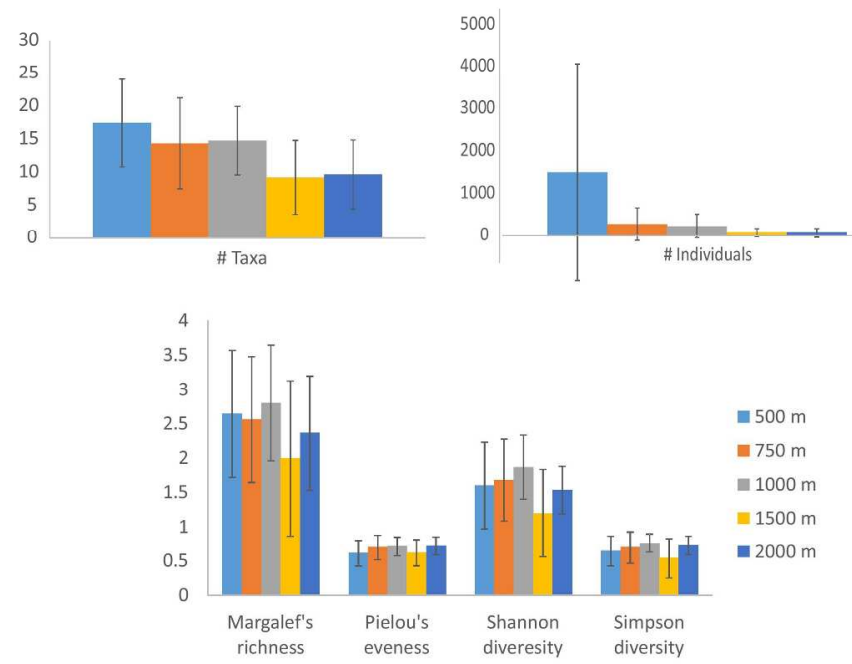

Figure 8. Average abundance and diversity indices from AGT data by depth with standard deviation. $209 \times 297 \mathrm{~mm}(300 \times 300$ DPI $)$ 
Figure 9. Average wet weight of VME taxa by phyla by set for both SUCS (upper) and AGT (lower). Box indicates sampled locations within the MPA. 
Figure 10. The relative abundance of large VME taxa averaged by SUCS set, size of pie chart proportional to the abundance recorded.

$327 \times 222 \mathrm{~mm}(300 \times 300 \mathrm{DPI})$ 
Figure 11. The relative wet weight of large VME taxa averaged by SUCS set, size of pie chart proportional to the total wet weight recorded.

$327 \times 222 \mathrm{~mm}(300 \times 300 \mathrm{DPI})$ 